\title{
Improved upper bounds for the star discrepancy of digital nets in dimension 3
}

\author{
by \\ Friedrich Pillichshammer (Linz)
}

1. Introduction. The concept of digital nets provides at the moment the most efficient method to generate point sets with small star discrepancy $D_{N}^{*}$. The star discrepancy of a set of points $\mathbf{x}_{0}, \ldots, \mathbf{x}_{N-1}$ in $[0,1)^{d}$ is defined by

$$
D_{N}^{*}=\sup _{B}\left|\frac{A_{N}(B)}{N}-\lambda(B)\right|
$$

where the supremum is taken over all subintervals $B$ of $[0,1)^{d}$ of the form $B=\prod_{i=1}^{d}\left[0, b_{i}\right), 0<b_{i} \leq 1, A_{N}(B)$ denotes the number of $i$ with $\mathbf{x}_{i} \in B$ and $\lambda$ is the Lebesgue measure.

A digital $(0, s, 3)$-net in base 2 is a set of $N=2^{s}$ points $\mathbf{x}_{0}, \ldots, \mathbf{x}_{N-1}$ in $[0,1)^{3}$ which is generated as follows: Choose three $s \times s$-matrices $C_{1}, C_{2}$ and $C_{3}$ over $\mathbb{Z}_{2}$ with the following property: For all integers $d_{1}, d_{2}, d_{3} \geq 0$ with $d_{1}+d_{2}+d_{3}=s$, the system of the first $d_{1}$ rows of $C_{1}$ together with the first $d_{2}$ rows of $C_{2}$ and the first $d_{3}$ rows of $C_{3}$ is linearly independent over $\mathbb{Z}_{2}$. Then to construct $\mathbf{x}_{n}:=\left(x_{n}^{(1)}, x_{n}^{(2)}, x_{n}^{(3)}\right)$ for $0 \leq n \leq 2^{s}-1$, represent $n$ in base 2 :

$$
n=n_{0}+n_{1} 2+\ldots+n_{s-1} 2^{s-1}
$$

with $n_{j} \in\{0,1\}$. Now multiply $C_{i}$ with the vector of digits:

$$
C_{i}\left(n_{0}, \ldots, n_{s-1}\right)^{\mathrm{T}}=:\left(y_{1}^{(i)}, \ldots, y_{s}^{(i)}\right)^{\mathrm{T}} \in \mathbb{Z}_{2}^{s}
$$

and set

$$
x_{n}^{(i)}:=\sum_{j=1}^{s} \frac{y_{j}^{(i)}}{2^{j}} .
$$

Further let us recall the definition of digital $(0,2)$-sequences in base 2: A digital $(0,2)$-sequence in base 2 is a sequence $\mathbf{x}_{0}, \mathbf{x}_{1}, \ldots$ in $[0,1)^{2}$ which is

2000 Mathematics Subject Classification: 11K06, 11K38, 42C10.

Key words and phrases: digital nets, digital sequence, star discrepancy, Walsh series analysis. 
generated as follows: Choose two $\mathbb{N} \times \mathbb{N}$-matrices $C_{1}$ and $C_{2}$ over $\mathbb{Z}_{2}$ such that for every integer $s \geq 1$ the upper left $s \times s$-matrices $C_{1}(s)$ and $C_{2}(s)$ generate a digital $(0, s, 2)$-net in base 2 (a digital $(0, s, 2)$-net in base 2 is defined analogously as a digital $(0, s, 3)$-net in base 2 - see Section 3$)$. Then to construct $\mathbf{x}_{n}:=\left(x_{n}^{(1)}, x_{n}^{(2)}\right)$ for $n \geq 0$, represent $n$ in base 2 :

$$
n=n_{0}+n_{1} 2+n_{2} 2^{2}+\ldots
$$

with $n_{j} \in\{0,1\}$. Now multiply $C_{i}$ with the vector of digits:

and set

$$
C_{i}\left(n_{0}, n_{1}, n_{2}, \ldots\right)^{\mathrm{T}}=:\left(y_{1}^{(i)}, y_{2}^{(i)}, \ldots\right)^{\mathrm{T}}
$$

$$
x_{n}^{(i)}:=\sum_{j=1}^{\infty} \frac{y_{j}^{(i)}}{2^{j}} .
$$

It was shown by H. Niederreiter in [6] that for the star discrepancy of any digital $(0, s, 3)$-net in base 2 we have

$$
N D_{N}^{*} \leq \frac{s^{2}}{4}+\frac{s}{2}+\frac{9}{4}
$$

and hence

$$
\limsup _{N \rightarrow \infty} \max \frac{N D_{N}^{*}}{(\log N)^{2}} \leq \frac{1}{4(\log 2)^{2}}=0.5203 \ldots,
$$

where the maximum is taken over all digital $(0, s, 3)$-nets in base 2 with $N=2^{s}$ elements.

Again in [6] Niederreiter proved that for the star discrepancy of the first $N$ elements of a digital $(0,2)$-sequence in base 2 we have

$$
N D_{N}^{*} \leq \frac{1}{8(\log 2)^{2}}(\log N)^{2}+\frac{11}{8 \log 2} \log N+\frac{9}{4}
$$

and hence

$$
\limsup _{N \rightarrow \infty} \max \frac{N D_{N}^{*}}{(\log N)^{2}} \leq \frac{1}{8(\log 2)^{2}}=0.26017 \ldots,
$$

where the maximum is taken over all digital $(0,2)$-sequences in base 2 . From this result he concluded for every integer $s \geq 1$ the existence of a digital $(0, s, 3)$-net in base 2 such that

$$
N D_{N}^{*} \leq s^{2} / 8+\mathcal{O}(s),
$$

where $N=2^{s}$.

In [1] H. Faure constructed a digital $(0,2)$-sequence in base 2 such that

$$
\limsup _{N \rightarrow \infty} \frac{N D_{N}^{*}}{(\log N)^{2}} \geq \frac{1}{24(\log 2)^{2}}=0.0867 \ldots
$$

In this paper we study the star discrepancy of digital $(0, s, 3)$-nets in base 2 and of digital $(0,2)$-sequences in base 2 . With the help of Walsh 
series analysis we improve the general bound for the star discrepancy of digital $(0, s, 3)$-nets in base 2 given by Niederreiter (Theorem 1). Further we give an improved upper bound for the star discrepancy of digital $(0,2)$ sequences in base 2 (Theorem 3 ) from which we conclude - in the same way as Niederreiter did in [6] - the existence of digital $(0, s, 3)$-nets in base 2 with an essentially smaller bound for the star discrepancy than the general bound given in Theorem 1 (Theorem 2).

2. The results. We have the following general upper bound for the star discrepancy of digital $(0, s, 3)$-nets in base 2 . This bound improves the discrepancy bound given in [6].

Theorem 1. For all digital $(0, s, 3)$-nets in base 2 we have

$$
N D_{N}^{*} \leq s^{2} / 6+\mathcal{O}(s)
$$

where $N=2^{s}$.

The proof will be given in Section 4. From Theorem 1 we immediately get the following corollary:

Corollary 1. We have

$$
\limsup _{N \rightarrow \infty} \max \frac{N D_{N}^{*}}{(\log N)^{2}} \leq \frac{1}{6(\log 2)^{2}}=0.34689 \ldots,
$$

where the maximum is taken over all digital $(0, s, 3)$-nets in base 2 .

Actually we can prove the existence of digital $(0, s, 3)$-nets in base 2 with an essentially smaller constant at the leading term in the discrepancy bound as given in Theorem 1 . We have

Theorem 2. For every $s \geq 1$ there exists a digital $(0, s, 3)$-net in base 2 such that

$$
N D_{N}^{*} \leq s^{2} / 12+\mathcal{O}(s),
$$

where $N=2^{s}$.

The proof of this theorem will be given in Section 5 . The digital $(0, s, 3)$ nets in base 2 for which the discrepancy bound in Theorem 2 holds are obtained by setting $\mathbf{x}_{n}=\left(n / 2^{s}, \mathbf{y}_{n}\right), n=0, \ldots, 2^{s}-1$, where $\mathbf{y}_{n}$ is the $n$th element of a digital $(0,2)$-sequence in base 2 . We shall see that the above Theorem 2 is a consequence of the following theorem:

TheOREM 3. For the star discrepancy $D_{N}^{*}$ of the first $N$ elements of a digital $(0,2)$-sequence in base 2 we have

$$
N D_{N}^{*} \leq \frac{1}{12(\log 2)^{2}}(\log N)^{2}+\frac{89}{36 \log 2} \log N+\frac{33}{6} .
$$

The proof of this theorem will be given in Section 5 . Combining the result from Theorem 3 with the result of Faure [1] mentioned in Section 1 we obtain 
Corollary 2. We have

$$
\frac{1}{24(\log 2)^{2}} \leq \limsup _{N \rightarrow \infty} \max \frac{N D_{N}^{*}}{(\log N)^{2}} \leq \frac{1}{12(\log 2)^{2}},
$$

where the maximum is taken over all digital $(0,2)$-sequences in base 2 .

3. Notation and auxiliary results. For $0 \leq \alpha, \beta, \gamma \leq 1$ we consider the discrepancy function

$$
\Delta(\alpha, \beta, \gamma):=A_{N}([0, \alpha) \times[0, \beta) \times[0, \gamma))-N \alpha \beta \gamma
$$

for digital $(0, s, 3)$-nets $\mathbf{x}_{0}, \ldots, \mathbf{x}_{2^{s}-1}$ in base 2 (i.e. $\left.N=2^{s}\right)$.

Since the generating matrices $C_{1}, C_{2}$ and $C_{3}$ of a $(0, s, 3)$-net must be regular, and since multiplying $C_{1}, C_{2}$ and $C_{3}$ by a regular matrix $A$ does not change the point set (only its order) we may always assume that

$$
\begin{gathered}
C_{1}=\left(\begin{array}{ccccc}
1 & 0 & \ldots & 0 & 0 \\
0 & 1 & \ldots & 0 & 0 \\
\ldots & \ldots & \ldots & \ldots \\
0 & 0 & \ldots & 1 & 0 \\
0 & 0 & \ldots & 0 & 1
\end{array}\right), \quad C_{2}=\left(\begin{array}{cccc}
c_{1,1}^{2} & c_{1,2}^{2} & \ldots & c_{1, s}^{2} \\
c_{2,1}^{2} & c_{2,2}^{2} & \ldots & c_{2, s}^{2} \\
\ldots \ldots & \ldots \ldots \ldots & \ldots \\
c_{s, 1}^{2} & c_{s, 2}^{2} & \ldots & c_{s, s}^{2}
\end{array}\right)=:\left(\begin{array}{c}
\vec{c}_{1}^{2} \\
\vec{c}_{2}^{2} \\
\vdots \\
\vdots \\
\vec{c}_{s}^{2}
\end{array}\right), \\
C_{3}=\left(\begin{array}{cccc}
c_{1,1}^{3} & c_{1,2}^{3} & \ldots & c_{1, s}^{3} \\
c_{2,1}^{3} & c_{2,2}^{3} & \ldots & c_{2, s}^{3} \\
\ldots \ldots & \ldots \ldots \ldots \\
c_{s, 1}^{3} & c_{s, 2}^{3} & \ldots & c_{s, s}^{3}
\end{array}\right)=:\left(\begin{array}{c}
\vec{c}_{1}^{3} \\
\vec{c}_{2}^{3} \\
\vdots \\
\vdots \\
\vec{c}_{s}^{3}
\end{array}\right) .
\end{gathered}
$$

Assume that $\alpha, \beta$ and $\gamma$ are "s-bit", i.e.

$$
\alpha=\frac{\alpha_{1}}{2}+\ldots+\frac{\alpha_{s}}{2^{s}}, \quad \beta=\frac{\beta_{1}}{2}+\ldots+\frac{\beta_{s}}{2^{s}}, \quad \gamma=\frac{\gamma_{1}}{2}+\ldots+\frac{\gamma_{s}}{2^{s}},
$$

and let $\alpha^{\prime}, \beta^{\prime}$ and $\gamma^{\prime}$ be arbitrary with

$$
\alpha \leq \alpha^{\prime}<\alpha+\frac{1}{2^{s}}, \quad \beta \leq \beta^{\prime}<\beta+\frac{1}{2^{s}}, \quad \gamma \leq \gamma^{\prime}<\gamma+\frac{1}{2^{s}} .
$$

Then (since all coordinates of the points of a digital net are $s$-bit) we have

$$
\Delta\left(\alpha^{\prime}, \beta^{\prime}, \gamma^{\prime}\right)=\Delta(\alpha, \beta, \gamma)-2^{s}\left(\alpha^{\prime} \beta^{\prime} \gamma^{\prime}-\alpha \beta \gamma\right),
$$

and hence for the star-discrepancy $D_{N}^{*}$ of the net we have

$$
\left|D_{N}^{*}-\frac{1}{N} \max _{\substack{\alpha, \beta, \gamma \\ s \text {-bit }}}\right| \Delta(\alpha, \beta, \gamma)||<\frac{3}{N}-\frac{3}{N^{2}}+\frac{1}{N^{3}}
$$

(note that $N=2^{s}$ ). 
We will call

$$
\frac{1}{N} \max _{\substack{\alpha, \beta, \gamma \\ s \text {-bit }}}|\Delta(\alpha, \beta, \gamma)|=: D_{N}^{\mathrm{d}}
$$

the discrete discrepancy of the net. $D_{N}^{\mathrm{d}}$ differs from $D_{N}^{*}$ at most by the almost negligible quantity $3 / N$ and seems for nets to be the more natural measure for the irregularities of distribution.

We need some further notation: For any $s$-bit number $\delta=\delta_{1} / 2+\ldots+$ $\delta_{s} / 2^{s}$ we write

$$
\vec{\delta}:=\left(\begin{array}{c}
\delta_{1} \\
\delta_{2} \\
\vdots \\
\delta_{s}
\end{array}\right),
$$

and for a non-negative integer $k=k_{s-1} 2^{s-1}+\ldots+k_{1} 2+k_{0}$ we write

$$
\vec{k}:=\left(\begin{array}{c}
k_{0} \\
k_{1} \\
\vdots \\
k_{s-1}
\end{array}\right) .
$$

For the proof of Theorem 1 we need two auxiliary results.

Lemma 1. Let $z$ be of the form $z=p / 2^{s}, p \in\left\{0, \ldots, 2^{s}-1\right\}$ (i.e. $z$ is $s$-bit). Then for the characteristic function $\chi_{[0, z)}$ of the interval $[0, z)$ we have

$$
\chi_{[0, z)}(x)=\sum_{k=0}^{2^{s}-1} c_{k}(z) \operatorname{wal}_{k}(x),
$$

where wal $_{k}$ denotes the $k$ th Walsh function in base 2 (see Remark 1),

$$
c_{k}(z)= \begin{cases}z & \text { if } k=0, \\ \operatorname{wal}_{k}(z) \frac{1}{2^{v(k)}} \psi\left(2^{v(k)} z\right) & \text { if } k \neq 0,\end{cases}
$$

where $\psi(x)$ is periodic with period 1 and

$$
\psi(x)= \begin{cases}x & \text { if } 0 \leq x<1 / 2, \\ x-1 & \text { if } 1 / 2 \leq x<1\end{cases}
$$

and $v(k)=r$ if $2^{r} \leq k<2^{r+1}($ for $k=0$ define $v(0):=-1)$.

REMARK 1. Recall that Walsh functions in base 2 can be defined as follows: For a non-negative integer $k$ with base 2 representation $k=k_{m} 2^{m}+$ $\ldots+k_{1} 2+k_{0}$ and a real $x$ with (canonical) base 2 representation $x=$ $x_{1} / 2+x_{2} / 2^{2}+\ldots$ we have

$$
\operatorname{wal}_{k}(x)=(-1)^{x_{1} k_{0}+x_{2} k_{1}+\ldots+x_{m+1} k_{m}}=(-1)^{(\vec{k} \mid \vec{x})} .
$$


Proof of Lemma 1. This is a simple calculation, to be found for example in [3, Lemma 2].

Lemma 2. Let $\psi$ be as in Lemma 1. Then

$$
\psi\left(2^{l+1} \beta\right)-\sum_{i=0}^{l} \psi\left(2^{i} \beta\right)=\{\beta\}-\beta_{l+2},
$$

where $\{\beta\}=\beta-[\beta]$.

Proof. See [4, Lemma 2].

For the proof of Theorem 3 we need some further notation and auxiliary results:

The concept of shifted digital $(0, s, 2)$-nets in base 2 is a slight generalization of the well known concept of digital $(0, s, 2)$-nets in base 2 . A shifted digital $(0, s, 2)$-net in base 2 is a set of $N=2^{s}$ points $\mathbf{x}_{0}, \ldots, \mathbf{x}_{N-1}$ in $[0,1)^{2}$ which is generated as follows: Choose two $s \times s$-matrices $C_{1}, C_{2}$ over $\mathbb{Z}_{2}$ with the following property: For every integer $k, 0 \leq k \leq s$, the system of the first $k$ rows of $C_{1}$ together with the first $s-k$ rows of $C_{2}$ is linearly independent over $\mathbb{Z}_{2}$. Further choose two fixed vectors $\vec{k}_{i}=\left(k_{1}^{(i)}, \ldots, k_{s}^{(i)}\right)^{\mathrm{T}} \in \mathbb{Z}_{2}^{s}$, $i=1,2$. Then to construct $\mathbf{x}_{n}:=\left(x_{n}^{(1)}, x_{n}^{(2)}\right)$ for $0 \leq n \leq 2^{s}-1$, represent $n$ in base 2 :

$$
n=n_{0}+n_{1} 2+\ldots+n_{s-1} 2^{s-1}
$$

with $n_{j} \in\{0,1\}$. Now multiply $C_{i}$ with the vector of digits and add the vector $\vec{k}_{i}$, i.e.:

$$
C_{i}\left(n_{0}, \ldots, n_{s-1}\right)^{\mathrm{T}}+\vec{k}_{i}=:\left(y_{1}^{(i)}, \ldots, y_{s}^{(i)}\right)^{\mathrm{T}} \in \mathbb{Z}_{2}^{s}
$$

and set

$$
x_{n}^{(i)}:=\sum_{j=1}^{s} \frac{y_{j}^{(i)}}{2^{j}} .
$$

Remark 2. In the definition of usual digital $(0, s, 2)$-nets in base 2 the vectors $\vec{k}_{i}, i=1,2$, are omitted.

For the star discrepancy of shifted digital $(0, s, 2)$-nets in base 2 we have the following result:

Lemma 3. For the star discrepancy $D_{N}^{*}$ of a shifted digital $(0, s, 2)$-net in base 2 we have

$$
N D_{N}^{*} \leq \frac{s}{3}+\frac{19}{9}
$$

where $N=2^{s}$. 
Proof. In [4, Theorem 5] this lemma was proved for digital $(0, s, 2)$-nets in base 2. It easily follows from the proof that the assertion is also true for shifted digital nets.

Finally we need the following general result which is well known in the theory of uniform distribution modulo one:

LEMMA 4. Let $\mathbf{x}_{0}, \ldots, \mathbf{x}_{N-1}$ be a point set in $[0,1)^{d}$ with star discrepancy $D_{N}^{*}$. Let $\mathbf{x}_{n}:=\left(x_{n}^{(1)}, \ldots, x_{n}^{(d)}\right), 0 \leq n \leq N-1$, and let $\varepsilon_{n}^{(i)}, 0 \leq$ $n \leq N-1,1 \leq i \leq d$, be non-negative reals with $\varepsilon_{n}^{(i)}<1 / a$, such that $x_{n}^{(i)}+\varepsilon_{n}^{(i)}<1$, for all $0 \leq n \leq N-1,1 \leq i \leq d$. Then for the star discrepancy $\widetilde{D}_{N}^{*}$ of the point set $\widetilde{\mathbf{x}}_{0}, \ldots, \widetilde{\mathbf{x}}_{N-1}$, with $\widetilde{x}_{n}^{(i)}:=x_{n}^{(i)}+\varepsilon_{n}^{(i)}$ for all $0 \leq n \leq N-1,1 \leq i \leq d$, we have

$$
\left|D_{N}^{*}-\widetilde{D}_{N}^{*}\right| \leq d / a
$$

4. Proof of Theorem 1. Due to formula (1) it suffices to show that

$$
N D_{N}^{\mathrm{d}} \leq s^{2} / 6+\mathcal{O}(s)
$$

for all digital $(0, s, 3)$-nets in base 2 .

Let $I:=[0, \alpha) \times[0, \beta) \times[0, \gamma)$ with $\alpha, \beta$ and $\gamma s$-bit. Then for $\mathbf{y}=$ $\left(y^{(1)}, y^{(2)}, y^{(3)}\right) \in[0,1)^{3}$ by Lemma 1 we have

$$
\begin{aligned}
\chi_{I}(\mathbf{y})-\lambda(I)= & \sum_{\substack{k, l, m=0 \\
(k, l, m) \neq(0,0,0)}} c_{k}(\alpha) c_{l}(\beta) c_{m}(\gamma) \operatorname{wal}_{k}\left(y^{(1)}\right) \operatorname{wal}_{l}\left(y^{(2)}\right) \mathrm{wal}_{m}\left(y^{(3)}\right) \\
= & \alpha \sum_{\substack{l, m=0 \\
(l, m) \neq(0,0)}}^{2^{s}-1} c_{l}(\beta) c_{m}(\gamma) \mathrm{wal}_{l}\left(y^{(2)}\right) \operatorname{wal}_{m}\left(y^{(3)}\right) \\
& +\beta \sum_{\substack{k, m=0 \\
(k, m) \neq(0,0)}}^{2^{s}-1} c_{k}(\alpha) c_{m}(\gamma) \operatorname{wal}_{k}\left(y^{(1)}\right) \mathrm{wal}_{m}\left(y^{(3)}\right) \\
& +\gamma \sum_{\substack{k, l=0 \\
(k, l) \neq(0,0)}}^{2^{s}-1} c_{k}(\alpha) c_{l}(\beta) \mathrm{wal}_{k}\left(y^{(1)}\right) \mathrm{wal}_{l}\left(y^{(2)}\right) \\
& +\sum_{k, l, m=1}^{2^{s}-1} \operatorname{wal}_{k}(\alpha) \mathrm{wal}_{l}(\beta) \mathrm{wal}_{m}(\gamma) \frac{\psi\left(2^{v(k)} \alpha\right) \psi\left(2^{v(l)} \beta\right) \psi\left(2^{v(m)} \gamma\right)}{2^{v(k)} 2^{v(l)} 2^{v(m)}} \\
& \times \operatorname{wal}_{k}\left(y^{(1)}\right) \mathrm{wal}_{l}\left(y^{(2)}\right) \mathrm{wal}_{m}\left(y^{(3)}\right) .
\end{aligned}
$$

Let now $\mathbf{x}_{i}, i=0, \ldots, 2^{s}-1$, with $\mathbf{x}_{i}:=\left(x_{i}^{(1)}, x_{i}^{(2)}, x_{i}^{(3)}\right)$ be a digital $(0, s, 3)$ - 
net in base 2 . Then we have

$$
\begin{aligned}
\Delta(\alpha, \beta, \gamma)= & \alpha \sum_{\substack{l, m=0 \\
(l, m) \neq(0,0)}}^{2^{s}-1} c_{l}(\beta) c_{m}(\gamma) \sum_{i=0}^{2^{s}-1} \operatorname{wal}_{l}\left(x_{i}^{(2)}\right) \mathrm{wal}_{m}\left(x_{i}^{(3)}\right) \\
& +\beta \sum_{\substack{k, m=0 \\
(k, m) \neq(0,0)}}^{2^{s}-1} c_{k}(\alpha) c_{m}(\gamma) \sum_{i=0}^{2^{s}-1} \operatorname{wal}_{k}\left(x_{i}^{(1)}\right) \mathrm{wal}_{m}\left(x_{i}^{(3)}\right) \\
+ & \gamma \sum_{\substack{k, l=0 \\
(k, l) \neq(0,0)}}^{2^{s}-1} c_{k}(\alpha) c_{l}(\beta) \sum_{i=0}^{2^{s}-1} \mathrm{wal}_{k}\left(x_{i}^{(1)}\right) \mathrm{wal}_{l}\left(x_{i}^{(2)}\right) \\
& +\sum_{\substack{k, l, m=1 \\
2^{s}-1}}^{2^{s}-1} \operatorname{wal}_{k}(\alpha) \mathrm{wal}_{l}(\beta) \mathrm{wal}_{m}(\gamma) \frac{\psi\left(2^{v(k)} \alpha\right) \psi\left(2^{v(l)} \beta\right) \psi\left(2^{v(m)} \gamma\right)}{2^{v(k)} 2^{v(l)} 2^{v(m)}} \\
& \times \sum_{i=0} \operatorname{wal}_{k}\left(x_{i}^{(1)}\right) \mathrm{wal}_{l}\left(x_{i}^{(2)}\right) \operatorname{wal}_{m}\left(x_{i}^{(3)}\right) \\
= & \alpha \Sigma_{1}+\beta \Sigma_{2}+\gamma \Sigma_{3}+\Sigma_{4} .
\end{aligned}
$$

From $[4$, Theorem 5] together with the proof of $[4$, Theorem 1] it follows that

$$
\left|\Sigma_{i}\right| \leq \frac{s}{3}+\frac{19}{9}
$$

for $i=1,2,3$, and hence it suffices to show that

$$
\left|\Sigma_{4}\right| \leq s^{2} / 6+\mathcal{O}(s)
$$

for all digital $(0, s, 3)$-nets in base 2 .

We now consider $\sum_{i=0}^{2^{s}-1} \operatorname{wal}_{k}\left(x_{i}^{(1)}\right) \operatorname{wal}_{l}\left(x_{i}^{(2)}\right) \operatorname{wal}_{m}\left(x_{i}^{(3)}\right)$ with $x_{i}^{(1)}:=$ $x_{i, 1}^{(1)} / 2+\ldots+x_{i, s}^{(1)} / 2^{s}, x_{i}^{(2)}:=x_{i, 1}^{(2)} / 2+\ldots+x_{i, s}^{(2)} / 2^{s}$ and $x_{i}^{(3)}:=x_{i, 1}^{(3)} / 2+$ $\ldots+x_{i, s}^{(3)} / 2^{s}$. We identify $\left(x_{i}^{(1)}, x_{i}^{(2)}, x_{i}^{(3)}\right)$ with

$$
\left(x_{i, 1}^{(1)}, \ldots, x_{i, s}^{(1)}, x_{i, 1}^{(2)}, \ldots, x_{i, s}^{(2)}, x_{i, 1}^{(3)}, \ldots, x_{i, s}^{(3)}\right)^{\mathrm{T}} \in\left(\mathbb{Z}_{2}\right)^{3 s}
$$

and we define

$$
\left(x_{i}^{(1)}, x_{i}^{(2)}, x_{i}^{(3)}\right) \oplus\left(\widetilde{x}_{i}^{(1)}, \widetilde{x}_{i}^{(2)}, \widetilde{x}_{i}^{(3)}\right):=\left(x_{i, 1}^{(1)}+\widetilde{x}_{i, 1}^{(1)}, \ldots, x_{i, s}^{(3)}+\widetilde{x}_{i, s}^{(3)}\right) .
$$

Further $\operatorname{wal}_{k, l, m}\left(x_{i}^{(1)}, x_{i}^{(2)}, x_{i}^{(3)}\right):=\operatorname{wal}_{k}\left(x_{i}^{(1)}\right) \operatorname{wal}_{l}\left(x_{i}^{(2)}\right) \operatorname{wal}_{m}\left(x_{i}^{(3)}\right)$, hence

$$
\begin{aligned}
\operatorname{wal}_{k, l, m}\left(\left(x_{i}^{(1)}, x_{i}^{(2)},\right.\right. & \left.\left.x_{i}^{(3)}\right) \oplus\left(\widetilde{x}_{i}^{(1)}, \widetilde{x}_{i}^{(2)}, \widetilde{x}_{i}^{(3)}\right)\right) \\
& =\operatorname{wal}_{k, l, m}\left(x_{i}^{(1)}, x_{i}^{(2)}, x_{i}^{(3)}\right) \operatorname{wal}_{k, l, m}\left(\widetilde{x}_{i}^{(1)}, \widetilde{x}_{i}^{(2)}, \widetilde{x}_{i}^{(3)}\right),
\end{aligned}
$$

i.e. $\operatorname{wal}_{k, l, m}$ is a character on $\left(\left(\mathbb{Z}_{2}\right)^{3 s}, \oplus\right)$. 
The digital net $\mathbf{x}_{0}, \ldots, \mathbf{x}_{2^{s}-1}$ is a subgroup of $\left(\left(\mathbb{Z}_{2}\right)^{3 s}, \oplus\right)$, hence

$$
\sum_{i=0}^{2^{s}-1} \operatorname{wal}_{k}\left(x_{i}^{(1)}\right) \operatorname{wal}_{l}\left(x_{i}^{(2)}\right) \operatorname{wal}_{m}\left(x_{i}^{(3)}\right)= \begin{cases}2^{s} \quad & \text { if } \operatorname{wal}_{k, l, m}\left(x_{i}^{(1)}, x_{i}^{(2)}, x_{i}^{(3)}\right)=1 \\ & \text { for all } i=0, \ldots, 2^{s}-1, \\ 0 & \text { otherwise }\end{cases}
$$

(For more details see [2] or [5].)

Now we have $\operatorname{wal}_{k, l, m}\left(x_{i}^{(1)}, x_{i}^{(2)}, x_{i}^{(3)}\right)=(-1)^{\left(\vec{k} \mid \vec{x}_{i}^{(1)}\right)+\left(\vec{l} \mid \vec{x}_{i}^{(2)}\right)+\left(\vec{m} \mid \vec{x}_{i}^{(3)}\right)}=1$ for all $i=0, \ldots, 2^{s}-1$ iff

$$
\left(\vec{k} \mid \vec{x}_{i}^{(1)}\right)=\left(\vec{l} \mid \vec{x}_{i}^{(2)}\right)+\left(\vec{m} \mid \vec{x}_{i}^{(3)}\right) \quad \text { for all } i=0, \ldots, 2^{s}-1
$$

(by the definition of the net); this means

$$
(\vec{k} \mid \vec{i})=\left(\vec{l} \mid C_{2} \vec{i}\right)+\left(\vec{m} \mid C_{3} \vec{i}\right) \quad \text { for all } i=0, \ldots, 2^{s}-1,
$$

and this is satisfied if and only if

$$
\vec{k}=C_{2}^{\mathrm{T}} \vec{l}+C_{3}^{\mathrm{T}} \vec{m}=: \vec{k}(l, m) .
$$

Further

$$
\operatorname{wal}_{k(l, m), l, m}(\alpha, \beta, \gamma)=\operatorname{wal}_{l}(\delta) \operatorname{wal}_{m}(\varepsilon)
$$

with $\vec{\delta}:=C_{2} \vec{\alpha}+\vec{\beta}$ and $\vec{\varepsilon}:=C_{3} \vec{\alpha}+\vec{\gamma}$ (note that $\delta_{i}=\left(\vec{c}_{i}^{2} \mid \vec{\alpha}\right)+\beta_{i}$ and $\left.\varepsilon_{i}=\left(\vec{c}_{i}^{3} \mid \vec{\alpha}\right)+\gamma_{i}\right)$.

Therefore we have

$$
\begin{aligned}
\Sigma_{4}= & 2^{s} \sum_{\substack{l, m=1 \\
k(l, m) \neq 0}}^{2^{s}-1} \operatorname{wal}_{l}(\delta) \operatorname{wal}_{m}(\varepsilon) \frac{\psi\left(2^{v(k(l, m))} \alpha\right) \psi\left(2^{v(l)} \beta\right) \psi\left(2^{v(m)} \gamma\right)}{2^{v(k(l, m))+v(l)+v(m)}} \\
= & 2^{s} \sum_{u, v=0}^{s-1} \frac{\psi\left(2^{u} \beta\right) \psi\left(2^{v} \gamma\right)}{2^{u+v}} \\
& \times \underbrace{\sum_{l=2^{u}}^{2^{u+1}-1} \sum_{m=2^{v}}^{2^{v+1}-1}}_{k(l, m) \neq 0} \operatorname{wal}_{l}(\delta) \mathrm{wal}_{m}(\varepsilon) \frac{\psi\left(2^{v(k(l, m))} \alpha\right)}{2^{v(k(l, m))}} .
\end{aligned}
$$

For $2^{u} \leq l \leq 2^{u+1}-1,2^{v} \leq m \leq 2^{v+1}-1$ we have

$$
\begin{aligned}
\operatorname{wal}_{l}(\delta) \operatorname{wal}_{m}(\varepsilon)= & (-1)^{l_{0} \delta_{1}+\ldots+l_{u-1} \delta_{u}+\delta_{u+1}}(-1)^{m_{0} \varepsilon_{1}+\ldots+m_{v-1} \varepsilon_{v}+\varepsilon_{v+1}} \\
= & (-1)^{l_{0} \delta_{1}+\ldots+l_{u-1} \delta_{u}+m_{0} \varepsilon_{1}+\ldots+m_{v-1} \varepsilon_{v}} \\
& \times(-1)^{\left(\vec{c}_{u+1}^{2} \mid \vec{\alpha}\right)+\left(\vec{c}_{v+1}^{3} \mid \vec{\alpha}\right)+\beta_{u+1}+\gamma_{v+1}}
\end{aligned}
$$


by the definition of $\delta$ and $\varepsilon$. Hence

$$
\begin{aligned}
\Sigma_{4}= & \sum_{u, v=0}^{s-1} \frac{\left\|2^{u} \beta\right\| \cdot\left\|2^{v} \gamma\right\|}{2^{u+v-s}}(-1)^{\left(\vec{c}_{u+1}^{2}+\vec{c}_{v+1}^{3} \mid \vec{\alpha}\right)} \\
& \times \underbrace{\sum_{l=2^{u}}^{2^{u+1}-1} \sum_{m=2^{v}}^{2^{v+1}-1}}_{k(l, m) \neq 0}(-1)^{l_{0} \delta_{1}+\ldots+l_{u-1} \delta_{u}+m_{0} \varepsilon_{1}+\ldots+m_{v-1} \varepsilon_{v}} \frac{\psi\left(2^{v(k(l, m))} \alpha\right)}{2^{v(k(l, m))}} .
\end{aligned}
$$

Here $l:=l_{0}+l_{1} 2+\ldots+l_{u} 2^{u}, m=m_{0}+m_{1} 2+\ldots+m_{v} 2^{v}$ and $\|\cdot\|$ is the distance to the nearest integer function, i.e. $\|x\|:=\min (x-[x], 1-(x-[x]))$. Note that $\psi\left(2^{u} \beta\right)(-1)^{\beta_{u+1}}=\left\|2^{u} \beta\right\|$ and $\psi\left(2^{v} \gamma\right)(-1)^{\gamma_{v+1}}=\left\|2^{v} \gamma\right\|$.

For $0 \leq u, v \leq s-1$ we have

$$
\begin{aligned}
\Sigma_{5}(u, v) & :=\underbrace{\sum_{l=2^{u}}^{2^{u+1}-1} \sum_{m=2^{v}}^{2^{v+1}-1}}_{k(l, m) \neq 0}(-1)^{l_{0} \delta_{1}+\ldots+l_{u-1} \delta_{u}+m_{0} \varepsilon_{1}+\ldots+m_{v-1} \varepsilon_{v}} \frac{\psi\left(2^{v(k(l, m))} \alpha\right)}{2^{v(k(l, m))}} \\
& =\sum_{w=0} \frac{\psi\left(2^{w} \alpha\right)}{2^{w}} \underbrace{\sum_{l=2^{u}} \sum_{m=2^{v}}}_{v(k(l, m))=w}(-1)^{l_{0} \delta_{1}+\ldots+l_{u-1} \delta_{u}+m_{0} \varepsilon_{1}+\ldots+m_{v-1} \varepsilon_{v}} .
\end{aligned}
$$

For $0 \leq u, v, w \leq s-1$ define

$$
\begin{aligned}
\Sigma_{6}(u, v, w) & :=\underbrace{\sum_{l=2^{u}}^{2^{u+1}-1} \sum_{m=2^{v}}^{2^{v+1}-1}}_{v(k(l, m))=w}(-1)^{l_{0} \delta_{1}+\ldots+l_{u-1} \delta_{u}+m_{0} \varepsilon_{1}+\ldots+m_{v-1} \varepsilon_{v}} \\
& =\underbrace{\sum_{l=0}^{2^{u}-1} \sum_{m=0}^{2^{v}-1}}_{v\left(k\left(l+2^{u}, m+2^{v}\right)\right)=w} \operatorname{wal}_{l}(\delta) \mathrm{wal}_{m}(\varepsilon) .
\end{aligned}
$$

For $0 \leq l \leq 2^{u}-1$ and $0 \leq m \leq 2^{v}-1$, the condition $v\left(k\left(l+2^{u}, m+2^{v}\right)\right)$ $=w$ means that there are $k_{0}, \ldots, k_{w-1} \in \mathbb{Z}_{2}$ such that

$$
\begin{aligned}
\vec{c}_{1}^{2} l_{0}+\ldots+\vec{c}_{u}^{2} l_{u-1}+\vec{c}_{u+1}^{2}+\vec{c}_{1}^{3} m_{0} & +\ldots+\vec{c}_{v}^{3} m_{v-1}+\vec{c}_{v+1}^{3} \\
& +\vec{e}_{1} k_{0}+\ldots+\vec{e}_{w} k_{w-1}+\vec{e}_{w+1}=\overrightarrow{0},
\end{aligned}
$$

where $\vec{e}_{i}$ is the $i$ th canonical vector in $\mathbb{Z}_{2}^{s}$ and $\overrightarrow{0}$ is the zero vector in $\mathbb{Z}_{2}^{s}$.

Since $\vec{c}_{1}^{2}, \ldots, \vec{c}_{u+1}^{2}, \vec{c}_{1}^{3}, \ldots, \vec{c}_{v+1}^{3}, \vec{e}_{1}, \ldots, \vec{e}_{w+1}$ by the $(0, s, 3)$-net property are linearly independent as long as $(u+1)+(v+1)+(w+1) \leq s$ we must have $u+v+w \geq s-2$. 
For $0 \leq l \leq 2^{u}-1$ and $0 \leq m \leq 2^{v}-1$, let

$$
\vec{n}:=\left(l_{0}, \ldots, l_{u-1}, m_{0}, \ldots, m_{v-1}\right)^{\mathrm{T}} \in \mathbb{Z}_{2}^{u+v}
$$

and define

$$
\vec{\zeta}:=\left(\delta_{1}, \ldots, \delta_{u}, \varepsilon_{1}, \ldots, \varepsilon_{v}\right)^{\mathrm{T}} \in \mathbb{Z}_{2}^{u+v} .
$$

Further let $\mathcal{C}^{(u, v)}$ be the $s \times(u+v)$-matrix over $\mathbb{Z}_{2}$ given by

$$
\mathcal{C}^{(u, v)}:=\left(\vec{c}_{1}^{2}, \ldots, \vec{c}_{u}^{2}, \vec{c}_{1}^{3}, \ldots, \vec{c}_{v}^{3}\right),
$$

and define

$$
\vec{d}=\vec{d}(u, v):=\vec{c}_{u+1}^{2}+\vec{c}_{v+1}^{3} \in \mathbb{Z}_{2}^{s} .
$$

Now (with this notation) $v\left(k\left(l+2^{u}, m+2^{v}\right)\right)=w$ means

$$
\mathcal{C}^{(u, v)} \vec{n}=\left(\begin{array}{c}
k_{0} \\
\vdots \\
k_{w-1} \\
1 \\
0 \\
\vdots \\
0
\end{array}\right)+\vec{d}
$$

for some $k_{i} \in \mathbb{Z}_{2}$ (therefore in the following we sometimes write $\left.v(k(n))=w\right)$.

Now we have to consider three cases:

1. $u+v+w=s-2$. Then the matrix $\left(\mathcal{C}^{(u, v)}, \vec{e}_{1}, \ldots, \vec{e}_{w}\right)$ has rank $s-2$ and therefore the system (2) has one or no solution.

2. $u+v+w=s-1$. Then the matrix $\left(\mathcal{C}^{(u, v)}, \vec{e}_{1}, \ldots, \vec{e}_{w}\right)$ has rank $s-1$ and therefore the system (2) has one or no solution.

3. $u+v+w \geq s$. Then the matrix $\left(\mathcal{C}^{(u, v)}, \vec{e}_{1}, \ldots, \vec{e}_{w}\right)$ has rank $s$ and therefore the system (2) has exactly $2^{u+v+w-s}$ solutions.

In the following we give the solutions of the system (2) in the above three cases and calculate the values of $\Sigma_{6}(u, v, w)$.

1. $u+v+w=s-2$. Since $\vec{e}_{1}, \ldots, \vec{e}_{w+1}, \vec{c}_{1}^{2}, \ldots, \vec{c}_{u+1}^{2}, \vec{c}_{1}^{3}, \ldots, \vec{c}_{v+1}^{3}$ are linearly dependent we can find some $\lambda_{1}^{1}, \ldots, \lambda_{w+1}^{1}, \lambda_{1}^{2}, \ldots, \lambda_{u+1}^{2}, \lambda_{1}^{3}, \ldots, \lambda_{v+1}^{3}$ $\in \mathbb{Z}_{2}$ not all zero such that

$$
\sum_{i=1}^{w+1} \lambda_{i}^{1} \vec{e}_{i}+\sum_{i=1}^{u+1} \lambda_{i}^{2} \vec{c}_{i}^{2}+\sum_{i=1}^{v+1} \lambda_{i}^{3} \vec{c}_{i}^{3}=\overrightarrow{0} .
$$

Assume that $\lambda_{w+1}^{1}=0$. Then $\vec{e}_{1}, \ldots, \vec{e}_{w}, \vec{c}_{1}^{2}, \ldots, \vec{c}_{u+1}^{2}, \vec{c}_{1}^{3}, \ldots, \vec{c}_{v+1}^{3}$ are linearly dependent. But this is a contradiction to the $(0, s, 3)$-net property and hence $\lambda_{w+1}^{1}=1$. In the same way one can show that $\lambda_{u+1}^{2}=1$ and $\lambda_{v+1}^{3}=1$ and hence the system (2) has exactly one solution. 
Now let $D=D(u, v)$ be the following $(u+v) \times(u+v)$-matrix over $\mathbb{Z}_{2}$ :

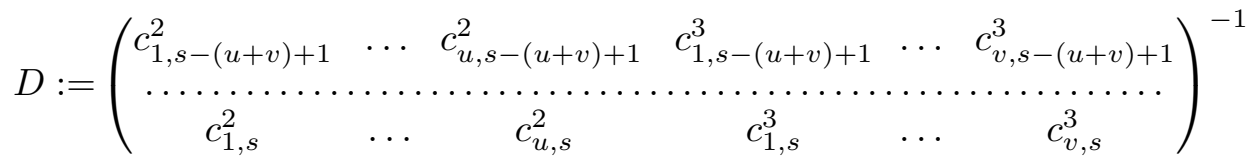
(note that $D=D(u, v)$ exists due to the $(0, s, 3)$-net property). We have

$$
\Sigma_{6}(u, v, w)=\sum_{\substack{n=0 \\ v(k(n))=w}}^{2^{u+v}-1}(-1)^{(\vec{n} \mid \vec{\zeta})}=\sum_{\substack{D n=0 \\ v(k(D n))=w}}^{2^{u+v}-1}(-1)^{(D \vec{n} \mid \vec{\zeta})}
$$

Now $v(k(D n))=w$ means that

$$
\mathcal{C}^{(u, v)} D \vec{n}=\left(\begin{array}{c}
k_{0} \\
\vdots \\
k_{w-1} \\
1 \\
0 \\
\vdots \\
0
\end{array}\right)+\vec{d}
$$

for some $k_{i} \in \mathbb{Z}_{2}$. This is equivalent to

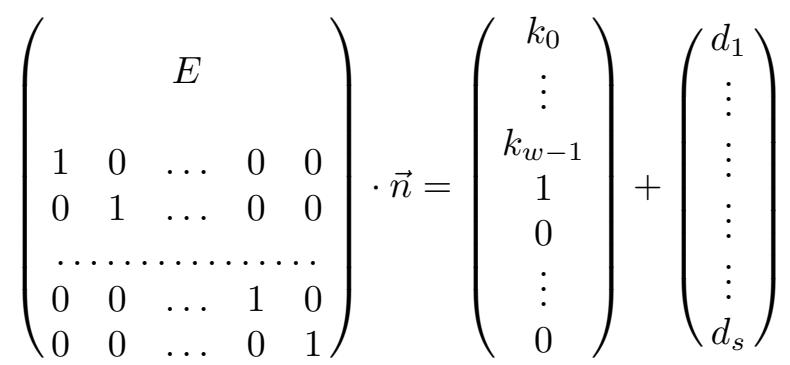

with

$$
E=\left(\begin{array}{ccc}
c_{1,1}^{2} & \cdots & c_{v, 1}^{3} \\
\ldots \ldots \cdots & \ldots & \ldots \\
c_{1, s-(u+v)}^{2} & \cdots & c_{v, s-(u+v)}^{3}
\end{array}\right) \cdot D
$$

i.e. an $(s-(u+v)) \times(u+v)$-matrix. Therefore the unique solution $\vec{n}$ is given by

$$
\vec{n}=\left(d_{s-(u+v)+1}, \ldots, d_{s}\right)^{\mathrm{T}} \in \mathbb{Z}_{2}^{u+v}
$$

and hence for $u+v+w=s-2$ we have

$$
\Sigma_{6}(u, v, w)=(-1)^{\left(\left(d_{s-(u+v)+1}, \ldots, d_{s}\right)^{\mathrm{T}} \mid D^{\mathrm{T}} \vec{\zeta}\right)} .
$$

2. $u+v+w=s-1$. Let the $(u+v) \times(u+v)$-matrix $D=D(u, v)$ be as in case 1 . We consider two subcases: 
(a) $u+v \leq s-2$. Assume that $D \vec{n}$ is a solution of the system (2). Then we find as in case 1 that

$$
\vec{n}=\left(d_{s-(u+v)+1}, \ldots, d_{s}\right)^{\mathrm{T}} \in \mathbb{Z}_{2}^{u+v} .
$$

Let $\vec{r} \in \mathbb{Z}_{2}^{u+v}$ be the last row of the $(s-(u+v)) \times(u+v)$-matrix

$$
E=\left(\begin{array}{ccc}
c_{1,1}^{2} & \ldots & c_{v, 1}^{3} \\
\ldots \ldots \ldots \ldots \ldots \ldots \ldots \ldots & \ldots \ldots \ldots \\
c_{1, s-(u+v)}^{2} & \ldots & c_{v, s-(u+v)}^{3}
\end{array}\right) \cdot D .
$$

Then $(\vec{r} \mid \vec{n})=1+d_{s-(u+v)}$; but that contradicts case 1 from which we have $(\vec{r} \mid \vec{n})=d_{s-(u+v)}$. Hence system (2) has no solution in this case.

(b) $u+v=s-1$ (hence $w=0)$. From $(u+1)+(v+1)=s+1$ we deduce that $\vec{c}_{1}^{2}, \ldots, \vec{c}_{u+1}^{2}, \vec{c}_{1}^{3}, \ldots, \vec{c}_{v+1}^{3}$ are linearly dependent. Hence we can find some $\lambda_{1}, \ldots, \lambda_{u+1}, \mu_{1}, \ldots, \mu_{v+1} \in \mathbb{Z}_{2}$ not all zero such that

$$
\sum_{i=1}^{u+1} \lambda_{i} \vec{c}_{i}^{2}+\sum_{i=1}^{v+1} \mu_{i} \vec{c}_{i}^{3}=\overrightarrow{0} .
$$

Assume $\lambda_{u+1}=0$. Then $\vec{c}_{1}^{2}, \ldots, \vec{c}_{u}^{2}, \vec{c}_{1}^{3}, \ldots, \vec{c}_{v+1}^{3}$ are linearly dependent, which contradicts the $(0, s, 3)$-net property. So $\lambda_{u+1}=1$ and analogously $\mu_{v+1}=1$. Hence there exists a vector $\vec{n}_{0} \in \mathbb{Z}_{2}^{u+v}$ such that

$$
\mathcal{C}^{(u, v)} \vec{n}_{0}=\vec{d}
$$

Now consider the following linear equation system:

$$
\left(\begin{array}{cccccc}
c_{1,2}^{2} & \ldots & c_{u, 2}^{2} & c_{1,2}^{3} & \ldots & c_{v, 2}^{3} \\
\ldots \ldots \ldots \ldots & \ldots \ldots \ldots \ldots \ldots \ldots \ldots \ldots \\
c_{1, s}^{2} & \ldots & c_{u, s}^{2} & c_{1, s}^{3} & \ldots & c_{v, s}^{3}
\end{array}\right) \cdot \vec{n}=\left(\begin{array}{c}
d_{2} \\
\vdots \\
d_{s}
\end{array}\right) .
$$

This system has a unique solution and this solution is $\vec{n}_{0}$. From this together with (3) it follows that the system

$$
\mathcal{C}^{(u, v)} \vec{n}=\vec{e}_{1}+\vec{d}
$$

cannot have a solution.

Altogether for $u+v+w=s-1$ we have

$$
\Sigma_{6}(u, v, w)=0 .
$$

3. $u+v+w \geq s$. We know that system (2) has exactly $2^{u+v+w-s}$ solutions. Again we consider two subcases.

(a) $u+v \leq s$. Let the $(u+v) \times(u+v)$-matrix $D=D(u, v)$ be like in case 1 . Proceeding as in case 1 we find that the solutions of system (2) are given by $D \vec{n}$ where

$$
\vec{n}=\left(n_{0}, \ldots, n_{u+v+w-(s+1)}, d_{w+1}+1, d_{w+2}, \ldots, d_{s}\right)^{\mathrm{T}} \in \mathbb{Z}_{2}^{s},
$$


with arbitrary $n_{0}, \ldots, n_{u+v+w-(s+1)} \in \mathbb{Z}_{2}$. From this we get

$$
\begin{aligned}
\Sigma_{6}(u, v, w)= & \sum_{\substack{D n=0 \\
v(k(D n))=w}}^{2^{u+v}-1}(-1)^{(D \vec{n} \mid \vec{\zeta})}=\sum_{\substack{n_{0}, \ldots, n_{u+v+w-(s+1)} \in \mathbb{Z}_{2} \\
\vec{n} \text { as in }(4)}}(-1)^{\left(\vec{n} \mid D^{\mathrm{T}} \vec{\zeta}\right)} \\
= & (-1)^{\left(\left(0, \ldots, 0, d_{w+1}+1, d_{w+2}, \ldots, d_{s}\right)^{\mathrm{T}} \mid D^{\mathrm{T}} \vec{\zeta}\right)} \sum_{n=0}^{2^{u+v+w-s}-1} \operatorname{wal}_{n}\left(D^{\mathrm{T}} \zeta\right) \\
= & 2^{u+v+w-s}(-1)^{\left(\left(0, \ldots, 0, d_{w+1}+1, d_{w+2}, \ldots, d_{s}\right)^{\mathrm{T}} \mid D^{\mathrm{T}} \vec{\zeta}\right)} \\
& \times \begin{cases}1 & \text { if }\left(D^{\mathrm{T}} \vec{\zeta} \mid \vec{e}_{i}\right)=0 \\
& \text { for all } i=1, \ldots, u+v+w-s, \\
0 & \text { otherwise. }\end{cases}
\end{aligned}
$$

Let $\left(D^{\mathrm{T}} \vec{\zeta} \mid \vec{e}_{i}\right)=0$ for all $i=1, \ldots, u+v+w-s$. Then

$$
\begin{aligned}
& \left(D^{\mathrm{T}} \vec{\zeta} \mid\left(0, \ldots, 0, d_{w+1}+1, d_{w+2}, \ldots, d_{s}\right)^{\mathrm{T}}\right) \\
& \quad=\left(D^{\mathrm{T}} \vec{\zeta} \mid\left(d_{s-(u+v)+1}, \ldots, d_{s}\right)^{\mathrm{T}}\right)+\left(D^{\mathrm{T}} \vec{\zeta} \mid \vec{e}_{u+v+w-s+1}\right) .
\end{aligned}
$$

Hence for $u+v+w \geq s, u+v \leq s$ we have

$$
\begin{aligned}
\Sigma_{6}(u, v, w)= & 2^{u+v+w-s}(-1)^{\left(D^{\mathrm{T}} \vec{\zeta} \mid\left(d_{s-(u+v)+1}, \ldots, d_{s}\right)^{\mathrm{T}}\right)} \\
& \times(-1)^{\left(D^{\mathrm{T}} \vec{\zeta} \mid \vec{e}_{u+v+w-s+1}\right)} \kappa_{1}(u, v, w, s)
\end{aligned}
$$

where

$$
\kappa_{1}(u, v, w, s)= \begin{cases}1 & \text { if }\left(D^{\mathrm{T}} \vec{\zeta} \mid \vec{e}_{i}\right)=0 \text { for all } i=1, \ldots, u+v+w-s \\ 0 & \text { otherwise. }\end{cases}
$$

(b) $u+v>s$. Let $F=F(u, v)$ be the following $s \times s$-matrix over $\mathbb{Z}_{2}$ :

$$
F=\left(\vec{c}_{1}^{2}, \ldots, \vec{c}_{u}^{2}, \vec{c}_{1}^{3}, \ldots, \vec{c}_{s-u}^{3}\right)^{-1}
$$

(note that $F$ exists due to the $(0, s, 3)$-net property) and let $G=G(u, v)$ be the following $(u+v) \times(u+v)$-matrix over $\mathbb{Z}_{2}$ :

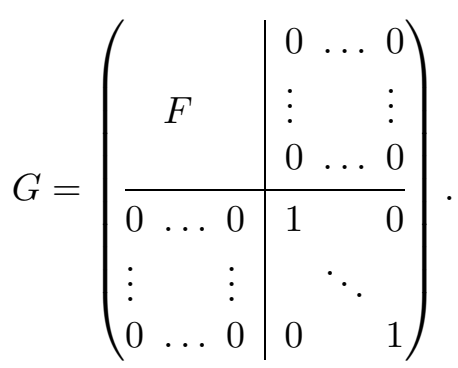


We have

$$
\Sigma_{6}(u, v, w)=\sum_{\substack{n=0 \\ v(k(n))=w}}^{2^{u+v}-1}(-1)^{(\vec{n} \mid \vec{\zeta})}=\sum_{\substack{G n=0 \\ v(k(G n))=w}}^{2^{u+v}-1}(-1)^{(G \vec{n} \mid \vec{\zeta})}
$$

Now $v(k(G n))=w$ means that

$$
\mathcal{C}^{(u, v)} G \vec{n}=\left(\begin{array}{c}
k_{0} \\
\vdots \\
k_{w-1} \\
1 \\
0 \\
\vdots \\
0
\end{array}\right)+\vec{d}
$$

for some $k_{i} \in \mathbb{Z}_{2}$. Since

$$
\mathcal{C}^{(u, v)} G=\left(I, \vec{c}_{s-u+1}^{3}, \ldots, \vec{c}_{v}^{3}\right)
$$

where $I$ is the $s \times s$ unit matrix, we get the following solutions for our equation system:

$$
\vec{n}=\left(\begin{array}{c}
\vec{d} \\
0 \\
\vdots \\
\vdots \\
0
\end{array}\right)+\left(\begin{array}{c}
k_{0} \\
\vdots \\
k_{w-1} \\
1 \\
0 \\
\vdots \\
0
\end{array}\right)+\sum_{i=1}^{u+v-s} r_{i} \cdot\left(\begin{array}{c}
\vec{c}_{s-u+i}^{3} \\
\vec{e}_{i}
\end{array}\right)
$$

for arbitrary $k_{i} \in \mathbb{Z}_{2}$ and arbitrary $r_{i} \in \mathbb{Z}_{2}$ and where $\vec{e}_{i}$ is the $i$ th unit vector in $\mathbb{Z}_{2}^{u+v-s}$.

Let $H=H(u, v)$ be the $(u+v) \times(u+v)$-matrix over $\mathbb{Z}_{2}$ given by

$$
H=\left(\begin{array}{lll|lll}
c_{s-u+1,1}^{3} & \ldots & c_{v, 1}^{3} & 0 & \ldots & 0 \\
\vdots & & \vdots & \vdots & & \vdots \\
c_{s-u+1, s}^{3} & \ldots & c_{v, s}^{3} & 0 & \ldots & 0 \\
\hline 1 & & 0 & 0 & \ldots & 0 \\
& \ddots & & \vdots & & \vdots \\
0 & & 1 & 0 & \ldots & 0
\end{array}\right) .
$$


Then we can write $\vec{n}$ as

$$
\vec{n}=\vec{e}_{w+1}+\left(\begin{array}{c}
\vec{d} \\
0 \\
\vdots \\
0
\end{array}\right)+\left(\begin{array}{c}
k_{0} \\
\vdots \\
k_{w-1} \\
0 \\
\vdots \\
0
\end{array}\right)+H \cdot\left(\begin{array}{c}
r_{1} \\
\vdots \\
r_{u+v-s} \\
0 \\
\vdots \\
0
\end{array}\right)
$$

where $\vec{e}_{w+1}$ is the $(w+1)$ th unit vector in $\mathbb{Z}_{2}^{u+v}$. Inserting in (5) yields

$$
\begin{aligned}
\Sigma_{6}(u, v, w)= & (-1)^{\left(\left(d_{1}, \ldots, d_{s}, 0, \ldots, 0\right)^{\mathrm{T}} \mid G^{\mathrm{T}} \vec{\zeta}\right)}(-1)^{\left(\vec{e}_{w+1} \mid G^{\mathrm{T}} \vec{\zeta}\right)} \\
& \times\left(\sum_{k_{0}, \ldots, k_{w-1} \in \mathbb{Z}_{2}}(-1)^{\left(\left(k_{0}, \ldots, k_{w-1}, 0, \ldots, 0\right)^{\mathrm{T}} \mid G^{\mathrm{T}} \vec{\zeta}\right)}\right) \\
& \times\left(\sum_{r_{1}, \ldots, r_{u+v-s} \in \mathbb{Z}_{2}}(-1)^{\left(H \cdot\left(r_{1}, \ldots, r_{u+v-s}, 0, \ldots, 0\right)^{\mathrm{T}} \mid G^{\mathrm{T}} \vec{\zeta}\right)}\right) \\
= & (-1)^{\left(\left(d_{1}, \ldots, d_{s}, 0, \ldots, 0\right)^{\mathrm{T}} \mid G^{\mathrm{T}} \vec{\zeta}\right)}(-1)^{\left(\vec{e}_{w+1} \mid G^{\mathrm{T}} \vec{\zeta}\right)} \\
& \times\left(\sum_{k=0}^{2^{w}-1} \operatorname{wal}_{k}\left(G^{\mathrm{T}} \zeta\right)\right)\left(\sum_{r=0}^{2^{u+v-s}-1} \operatorname{wal}_{r}\left(H^{\mathrm{T}} G^{\mathrm{T}} \zeta\right)\right) \\
= & (-1)^{\left(\left(d_{1}, \ldots, d_{s}, 0, \ldots, 0\right)^{\mathrm{T}} \mid G^{\mathrm{T}} \vec{\zeta}\right)}(-1)^{\left(\vec{e}_{w+1} \mid G^{\mathrm{T}} \vec{\zeta}\right)} \\
& \times 2^{u+v+w-s} \kappa_{2}(u, v, w, s) \kappa_{3}(u, v, s),
\end{aligned}
$$

where

$$
\begin{aligned}
\kappa_{2}(u, v, w, s) & = \begin{cases}1 & \text { if }\left(\vec{e}_{i} \mid G^{\mathrm{T}} \vec{\zeta}\right)=0 \text { for all } i=1, \ldots, w, \\
0 & \text { else },\end{cases} \\
\kappa_{3}(u, v, s) & = \begin{cases}1 & \text { if }\left(\vec{e}_{i} \mid H^{\mathrm{T}} G^{\mathrm{T}} \vec{\zeta}\right)=0 \text { for all } i=1, \ldots, u+v-s, \\
0 & \text { else. }\end{cases}
\end{aligned}
$$

Now we can evaluate $\Sigma_{5}(u, v)$ : We consider three cases.

1. $u+v>s$. Then

$$
\begin{aligned}
\Sigma_{5}(u, v)= & 2^{u+v-s}(-1)^{\left(\left(d_{1}, \ldots, d_{s}, 0, \ldots, 0\right)^{\mathrm{T}} \mid G^{\mathrm{T}} \vec{\zeta}\right)} \kappa_{3}(u, v, s) \\
& \times \sum_{w=0}^{s-1} \psi\left(2^{w} \alpha\right)(-1)^{\left(\vec{e}_{w+1} \mid G^{\mathrm{T}} \vec{\zeta}\right)} \kappa_{2}(u, v, w, s) .
\end{aligned}
$$

For $0 \leq u, v \leq s-1$ let

$$
m=m(u, v):=\max \left\{1 \leq j \leq u+v:\left(\vec{e}_{i} \mid G^{\mathrm{T}} \vec{\zeta}\right)=0, i=1, \ldots, j\right\}
$$

(if $u+v=0$ or if $\left(\vec{e}_{1} \mid G^{\mathrm{T}} \vec{\zeta}\right)=1$ set $m=m(u, v):=0$ ). By the definition of $m=m(u, v)$ we have $\left(\vec{e}_{1} \mid G^{\mathrm{T}} \vec{\zeta}\right)=\ldots=\left(\vec{e}_{m} \mid G^{\mathrm{T}} \vec{\zeta}\right)=0$ and $\left(\vec{e}_{m+1} \mid G^{\mathrm{T}} \vec{\zeta}\right)=1$. 
Hence $\kappa_{2}(u, v, w, s)=1$ iff $w \leq m(u, v)$. So we have

$$
\begin{aligned}
\Sigma_{5}(u, v)= & 2^{u+v-s}(-1)^{\left(\left(d_{1}, \ldots, d_{s}, 0, \ldots, 0\right)^{\mathrm{T}} \mid G^{\mathrm{T}} \vec{\zeta}\right)} \kappa_{3}(u, v, s) \\
& \times\left(\sum_{w=0}^{m-1} \psi\left(2^{w} \alpha\right)-\psi\left(2^{m} \alpha\right)\right) \\
= & 2^{u+v-s}(-1)^{\left(\left(d_{1}, \ldots, d_{s}, 0, \ldots, 0\right)^{\mathrm{T}} \mid G^{\mathrm{T}} \vec{\zeta}\right)} \kappa_{3}(u, v, s)\left(\alpha_{m+1}-\alpha\right),
\end{aligned}
$$

where we used Lemma 2. Hence

$$
\left|\Sigma_{5}(u, v)\right| \leq 2^{u+v-s}
$$

2. $u+v \leq s-2$. We have

$$
\begin{aligned}
\Sigma_{5}(u, v)= & \sum_{w=s-2-(u+v)}^{s-1} \frac{\psi\left(2^{w} \alpha\right)}{2^{w}} \Sigma_{6}(u, v, w) \\
= & \frac{\psi\left(2^{s-2-(u+v)} \alpha\right)}{2^{s-2-(u+v)} \Sigma_{6}(u, v, s-2-(u+v))} \\
& +\sum_{w=s-(u+v)}^{s-1} \frac{\psi\left(2^{w} \alpha\right)}{2^{w}} \Sigma_{6}(u, v, w) \\
= & 2^{u+v-s}(-1)^{\left(\left(d_{s-(u+v)+1}, \ldots, d_{s}\right)^{\mathrm{T}} \mid D^{\mathrm{T}} \vec{\zeta}\right)} \\
& \times\left[4 \psi\left(2^{s-2-(u+v)} \alpha\right)+\sum_{w=s-(u+v)}^{s-1} \psi\left(2^{w} \alpha\right)\right. \\
& \left.\times(-1)^{\left(\vec{e}_{u+v+w-s+1} \mid D^{\mathrm{T}} \vec{\zeta}\right)} \kappa_{1}(u, v, w, s)\right] .
\end{aligned}
$$

For $0 \leq u, v \leq s-1$ let

$$
p=p(u, v):=\max \left\{1 \leq j \leq u+v:\left(\vec{e}_{i} \mid D^{\mathrm{T}} \vec{\zeta}\right)=0, i=1, \ldots, j\right\}
$$

(if $u+v=0$ or if $\left(\vec{e}_{1} \mid D^{\mathrm{T}} \vec{\zeta}\right)=1$ set $p=p(u, v):=0$ ). By the definition of $p=p(u, v)$ we have

$$
\left(\vec{e}_{1} \mid G^{\mathrm{T}} \vec{\zeta}\right)=\ldots=\left(\vec{e}_{p} \mid G^{\mathrm{T}} \vec{\zeta}\right)=0 \quad \text { and } \quad\left(\vec{e}_{p+1} \mid G^{\mathrm{T}} \vec{\zeta}\right)=1 .
$$

Hence $\kappa_{1}(u, v, w, s)=1$ iff $u+v+w-s \leq p(u, v)$. So we have

$$
\begin{aligned}
\Sigma_{5}(u, v)= & 2^{u+v-s}(-1)^{\left(\left(d_{s-(u+v)+1}, \ldots, d_{s}\right)^{\mathrm{T}} \mid D^{\mathrm{T}} \vec{\zeta}\right)} \\
& \times\left[4 \psi\left(2^{s-2-(u+v)} \alpha\right)-\psi\left(2^{s-(u+v)+p} \alpha\right)+\sum_{w=s-(u+v)}^{s-(u+v)+p-1} \psi\left(2^{w} \alpha\right)\right] .
\end{aligned}
$$


Now with Lemma 2 we get

$$
\begin{aligned}
& \sum_{w=s-(u+v)}^{s-(u+v)+p-1} \psi\left(2^{w} \alpha\right)-\psi\left(2^{s-(u+v)+p} \alpha\right) \\
& =\sum_{w=0}^{s-(u+v)+p-1} \psi\left(2^{w} \alpha\right)-\psi\left(2^{s-(u+v)+p} \alpha\right)-\sum_{w=0}^{s-(u+v)-1} \psi\left(2^{w} \alpha\right) \\
& =\alpha_{s-(u+v)+p+1}-\alpha_{s-(u+v)+1}-\psi\left(2^{s-(u+v)} \alpha\right) .
\end{aligned}
$$

Moreover we have

$$
\begin{aligned}
4 \psi\left(2^{s-(u+v)-2} \alpha\right) & =4\left(\frac{\alpha_{s-(u+v)}}{2^{2}}+\ldots+\frac{\alpha_{s}}{2^{u+v+2}}-\frac{\alpha_{s-(u+v)-1}}{2}\right) \\
& =\alpha_{s-(u+v)}+\left\{2^{s-(u+v)} \alpha\right\}-2 \alpha_{s-(u+v)-1} .
\end{aligned}
$$

\section{Hence}

$$
\begin{aligned}
4 \psi\left(2^{s-2-(u+v)}\right)-\psi( & \left(2^{s-(u+v)+p} \alpha\right)+\sum_{w=s-(u+v)}^{s-(u+v)+p-1} \psi\left(2^{w} \alpha\right) \\
= & \alpha_{s-(u+v)}+\left\{2^{s-(u+v)} \alpha\right\}-2 \alpha_{s-(u+v)-1} \\
& \quad+\alpha_{s-(u+v)+p+1}-\alpha_{s-(u+v)+1}-\psi\left(2^{s-(u+v)} \alpha\right) \\
= & \alpha_{s-(u+v)+p+1}+\alpha_{s-(u+v)}-2 \alpha_{s-(u+v)-1} .
\end{aligned}
$$

Therefore we have

$$
\begin{aligned}
\Sigma_{5}(u, v)= & 2^{u+v-s}(-1)^{\left(\left(d_{s-(u+v)+1}, \ldots, d_{s}\right)^{\mathrm{T}} \mid D^{\mathrm{T}} \vec{\zeta}\right)} \\
& \times\left[\alpha_{s-(u+v)+p+1}+\alpha_{s-(u+v)}-2 \alpha_{s-(u+v)-1}\right] .
\end{aligned}
$$

Hence

$$
\left|\Sigma_{5}(u, v)\right| \leq 2 \cdot 2^{u+v-s} .
$$

3. $s-1 \leq u+v \leq s$. Then we get, as in case 2 ,

$$
\begin{aligned}
\Sigma_{5}(u, v)= & 2^{u+v-s}(-1)^{\left(\left(d_{s-(u+v)+1}, \ldots, d_{s}\right)^{\mathrm{T}} \mid D^{\mathrm{T}} \vec{\zeta}\right)} \\
& \times\left[\alpha_{s-(u+v)+p+1}-\alpha_{s-(u+v)+1}-\psi\left(2^{s-(u+v)} \alpha\right)\right] .
\end{aligned}
$$

From

$$
\begin{aligned}
\psi\left(2^{s-(u+v)} \alpha\right) & =\frac{\alpha_{s-(u+v)+2}}{2^{2}}+\ldots+\frac{\alpha_{s}}{2^{u+v}}-\frac{\alpha_{s-(u+v)+1}}{2} \\
& =\frac{1}{2}\left(\left\{2^{s-(u+v)+1} \alpha\right\}-\alpha_{s-(u+v)+1}\right)
\end{aligned}
$$

we get 


$$
\begin{aligned}
\mid \alpha_{s-(u+v)+p+1}- & \alpha_{s-(u+v)+1}-\psi\left(2^{s-(u+v)} \alpha\right) \mid \\
& =\left|\alpha_{s-(u+v)+p+1}-\frac{1}{2}\left(\alpha_{s-(u+v)+1}+\left\{2^{s-(u+v)+1} \alpha\right\}\right)\right| \leq 1
\end{aligned}
$$

and hence

$$
\left|\Sigma_{5}(u, v)\right| \leq 2^{u+v-s}
$$

Summing up we have

$$
\left|\Sigma_{5}(u, v)\right| \leq \begin{cases}2 \cdot 2^{u+v-s} & \text { for } u+v \leq s-2 \\ 2^{u+v-s} & \text { for } u+v \geq s-1\end{cases}
$$

Therefore

$$
\begin{aligned}
\left|\Sigma_{4}\right| & \leq \sum_{u, v=0}^{s-1} \frac{\left\|2^{u} \beta\right\| \cdot\left\|2^{v} \gamma\right\|}{2^{u+v-s}}\left|\Sigma_{5}(u, v)\right| \\
& \leq \sum_{u, v=0}^{s-1}\left\|2^{u} \beta\right\| \cdot\left\|2^{v} \gamma\right\|+\sum_{\substack{u, v=0 \\
u+v \leq s-2}}^{s-1}\left\|2^{u} \beta\right\| \cdot\left\|2^{v} \gamma\right\| .
\end{aligned}
$$

From [4, Theorem 3] we get

$$
\sum_{u, v=0}^{s-1}\left\|2^{u} \beta\right\| \cdot\left\|2^{v} \gamma\right\| \leq\left(\frac{s}{3}+\frac{1}{9}-(-1)^{s} \frac{1}{9 \cdot 2^{s}}\right)^{2} .
$$

Further, by [4, Theorem 2], we have

$$
\begin{aligned}
\sum_{\substack{u, v=0 \\
u+v \leq s-2}}^{s-1}\left\|2^{u} \beta\right\| \cdot\left\|2^{v} \gamma\right\| & =\sum_{u=0}^{s-1}\left\|2^{u} \beta\right\| \sum_{v=0}^{s-u-2}\left\|2^{v} \gamma\right\| \\
& \leq \sum_{u=0}^{s-1}\left\|2^{u} \beta\right\|\left(\frac{s-u-1}{3}+\frac{1}{9}-\frac{(-1)^{s-u-1}}{9 \cdot 2^{s-u-1}}\right)
\end{aligned}
$$

and

$$
\begin{aligned}
\sum_{u=0}^{s-1}\left\|2^{u} \beta\right\| \frac{s-u-1}{3} & \leq \frac{1}{6}+\frac{1}{3} \sum_{k=1}^{s-2} \sum_{u=0}^{s-k-1}\left\|2^{u} \beta\right\| \\
& \leq \frac{1}{6}+\frac{1}{3} \sum_{k=1}^{s-2}\left(\frac{s-k}{3}+\frac{1}{9}-\frac{(-1)^{s-k}}{9 \cdot 2^{s-k}}\right) \\
& =\frac{s^{2}}{18}-\frac{s}{54}-\frac{4}{162}+\frac{(-1)^{s}}{162 \cdot 2^{s-2}} .
\end{aligned}
$$


Together we have

$$
\begin{aligned}
\left|\Sigma_{4}\right| \leq & \left(\frac{s}{3}+\frac{1}{9}-(-1)^{s} \frac{1}{9 \cdot 2^{s}}\right)^{2}+\frac{s^{2}}{18}-\frac{s}{54}-\frac{4}{162}+\frac{(-1)^{s}}{162 \cdot 2^{s-2}} \\
& +\frac{2}{9}\left(\frac{s}{3}+\frac{1}{9}-(-1)^{s} \frac{1}{9 \cdot 2^{s}}\right) \\
= & \frac{s^{2}}{6}+s \cdot\left(\frac{7}{54}-\frac{2(-1)^{s}}{27 \cdot 2^{s}}\right)+\frac{1}{81}-\frac{2(-1)^{s}}{81 \cdot 2^{s}}+\frac{1}{81 \cdot 2^{2 s}}
\end{aligned}
$$

and the result follows.

\section{Proof of Theorems 2 and 3}

Proof of Theorem 3. We use the technique of Niederreiter introduced in [6, Proof of Lemma 4.1] (or see [7, Proof of Lemma 4.11]) and an idea of G. Larcher.

Let $N=b_{0}+b_{1} 2+\ldots+b_{r} 2^{r}$, with $b_{r}=1$ and $b_{k} \in\{0,1\}, 0 \leq k<r$, be the base 2 representation of $N$ and let the integer $p$ be maximal such that $2^{p}$ is a divisor of $N$.

Let the digital $(0,2)$-sequence in base 2 be generated by the $\mathbb{N} \times \mathbb{N}$ matrices $C_{1}$ and $C_{2}$. Divide the sequence $\mathbf{x}_{0}, \ldots, \mathbf{x}_{N-1}$ into subsequences $\omega_{m, b}$ for $b=0, \ldots, b_{m}-1$ and $m=0, \ldots, r$, where $\omega_{m, b}$ is the subsequence $\mathbf{x}_{n}$ with $\sum_{k=m+1}^{r} b_{k} 2^{k}+b 2^{m} \leq n<\sum_{k=m+1}^{r} b_{k} 2^{k}+(b+1) 2^{m}$. For fixed $m$ divide the matrices $C_{i}, i=1,2$, into the following parts:

$$
C_{i}=\left(\begin{array}{l|l}
C_{i}(m) & D_{i}(m) \\
\hline E_{i}(m)
\end{array}\right),
$$

where $C_{i}(m)$ is the upper left $m \times m$-submatrix of $C_{i}$. If

$$
n=\sum_{k=m+1}^{r} b_{k} 2^{k}+b 2^{m}+\sum_{k=0}^{m-1} a_{k} 2^{k},
$$

then

$$
\vec{n}=\left(a_{0}, a_{1}, \ldots, a_{m-1}, b, b_{m+1}, \ldots, b_{r}, 0,0, \ldots\right)^{\mathrm{T}}
$$

and 


$$
C_{i} \vec{n}=\left(\begin{array}{c}
a_{0} \\
a_{1} \\
\vdots \\
\vdots \\
a_{m-2}(m) \cdot \\
a_{m-1}
\end{array}\right)+\left(\begin{array}{c}
b \\
0 \\
\vdots
\end{array}\right)+\left(\begin{array}{c}
b_{m+1} \\
\vdots \\
b_{r} \\
0 \\
\vdots \\
0 \\
0 \\
\vdots
\end{array}\right)+\left(\begin{array}{c}
0 \\
0 \\
\vdots \\
\vdots \\
\vdots \\
0 \\
E_{i}(m) \vec{n}
\end{array}\right) .
$$

Hence $\omega_{m, b}$ is a modulo $\mathbb{Z}_{2}$ shifted digital $(0, m, 2)$-net in base 2 generated by $C_{1}(m)$ and $C_{2}(m)$, which finally is translated by a vector with positive coordinates less than $2^{-m}$. Let $\widetilde{\omega}_{m, b}$ be the shifted digital net without the final translation. Let $D_{m, b}^{*}$ (resp. $\widetilde{D}_{m, b}^{*}$ ) be the star discrepancy of $\omega_{m, b}$ (resp. $\left.\widetilde{\omega}_{m, b}\right)$. Then by Lemma 4 we have

$$
\left|D_{m, b}^{*}-\widetilde{D}_{m, b}^{*}\right| \leq \frac{2}{2^{m}} .
$$

Therefore we get, by Lemma 3 ,

$$
\begin{aligned}
N D_{N}^{*} & \leq \sum_{m=0}^{r} \sum_{b=0}^{b_{m}-1} 2^{m} D_{m, b}^{*} \leq \sum_{m=0}^{r} \sum_{b=0}^{b_{m}-1} 2^{m}\left(\frac{2}{2^{m}}+\widetilde{D}_{m, b}^{*}\right) \\
& \leq 2 \sum_{m=0}^{r} b_{m}+\sum_{m=0}^{r} b_{m}\left(\frac{m}{3}+\frac{19}{9}\right) \\
& =2 \sum_{m=p}^{r} b_{m}+\sum_{m=p}^{r} b_{m}\left(\frac{m}{3}+\frac{19}{9}\right),
\end{aligned}
$$

where $p$ is the maximal integer such that $2^{p}$ is a divisor of $N$.

Now apply the same method to the set consisting of the $\mathbf{x}_{n}$ with $N \leq$ $n \leq 2^{r+1}-1$. This set consists of $2^{r+1}-N$ points. Let

$$
2^{r+1}-N=\sum_{m=0}^{r} c_{m} 2^{m},
$$

with $c_{m} \in\{0,1\}$. Again we can split up this set into a union of subsequences. Let $\omega_{m, c}$ for $c=0, \ldots, c_{m}-1$ and $m=0, \ldots, r$ be the subsequence $\mathbf{x}_{n}$ with $2^{r+1}-\sum_{k=m+1}^{r} c_{k} 2^{k}-c 2^{m} \leq n<2^{r+1}-\sum_{k=m+1}^{r} c_{k} 2^{k}-c 2^{m}+2^{m}$. As above one can see that $\omega_{m, c}$ is a modulo $\mathbb{Z}_{2}$ shifted digital $(0, m, 2)$-net in base 2 generated by $C_{1}(m)$ and $C_{2}(m)$, which finally is translated by a vector with positive coordinates less than $2^{-m}$. As above, for the star discrepancy of our 
set we get

$$
\left(2^{r+1}-N\right) D_{2^{r+1}-N}^{*} \leq 2 \sum_{m=0}^{r} c_{m}+\sum_{m=0}^{r} c_{m}\left(\frac{m}{3}+\frac{19}{9}\right) .
$$

The first $2^{r+1}$ points of the (0,2)-sequence build a digital $(0, r+1,2)$-net. Our initial set is the difference between this $(0, r+1,2)$-net and the set of $\mathbf{x}_{n}$ with $N \leq n \leq 2^{r+1}-1$. Hence

$$
N D_{N}^{*} \leq 2 \sum_{m=0}^{r} c_{m}+\sum_{m=0}^{r} c_{m}\left(\frac{m}{3}+\frac{19}{9}\right)+\left(\frac{r+1}{3}+\frac{19}{9}\right) .
$$

Now

$$
2^{r+1}=2^{r+1}-N+N=\sum_{m=0}^{r}\left(c_{m}+b_{m}\right) 2^{m} .
$$

Hence we have $c_{0}=\ldots=c_{p-1}=0, b_{p}+c_{p}=2$ and $b_{m}+c_{m}=1$ for $m=p+1, \ldots, r$. Therefore

$$
\begin{aligned}
N D_{N}^{*} \leq & 2\left(2-b_{p}+\sum_{m=p+1}^{r}\left(1-b_{m}\right)\right)+\left(2-b_{p}\right)\left(\frac{p}{3}+\frac{19}{9}\right) \\
& +\sum_{m=p+1}^{r}\left(1-b_{m}\right)\left(\frac{m}{3}+\frac{19}{9}\right)+\left(\frac{r+1}{3}+\frac{19}{9}\right) .
\end{aligned}
$$

Hence

$$
\begin{aligned}
N D_{N}^{*} \leq \min \{ & 2 \sum_{m=p}^{r} b_{m}+\sum_{m=p}^{r} b_{m}\left(\frac{m}{3}+\frac{19}{9}\right) \\
& 2\left(1+\sum_{m=p}^{r}\left(1-b_{m}\right)\right)+\left(\frac{p}{3}+\frac{19}{9}\right) \\
& \left.+\sum_{m=p}^{r}\left(1-b_{m}\right)\left(\frac{m}{3}+\frac{19}{9}\right)+\left(\frac{r+1}{3}+\frac{19}{9}\right)\right\} .
\end{aligned}
$$

Now, since $\min (A, B) \leq(A+B) / 2$, the result follows.

Finally we give the proof of Theorem 2, which is an easy consequence of Theorem 3.

Proof of Theorem 2. Let $\mathbf{x}_{0}, \mathbf{x}_{1}, \ldots$ be a digital $(0,2)$-sequence in base 2 (such a sequence exists by [6, Corollary 6.19]) and let $s \geq 1$ be an integer. Then the set of

$$
\mathbf{y}_{n}:=\left(\frac{n}{2^{s}}, \mathbf{x}_{n}\right), \quad n=0, \ldots, 2^{s}-1
$$


is a digital $(0, s, 3)$-net in base 2 . For the star discrepancy of this net, by [6, Lemma 8.9] and by Theorem 3 we have

$$
N D_{N}^{*} \leq \frac{1}{12(\log 2)^{2}}(\log N)^{2}+\mathcal{O}(\log N),
$$

where $N=2^{s}$, and the result follows.

Acknowledgments. The author would like to thank Gerhard Larcher for valuable discussions and suggestions.

\section{References}

[1] H. Faure, Discrepancy lower bound in two dimensions, in: Monte Carlo and QuasiMonte Carlo Methods in Scientific Computing, Lecture Notes in Statist. 106, Springer, 1995, 198-204.

[2] G. Larcher, H. Niederreiter and W. Ch. Schmid, Digital nets and sequences constructed over finite rings and their application to quasi-Monte Carlo integration, Monatsh. Math. 121 (1996), 231-253.

[3] G. Larcher and F. Pillichshammer, Walsh series analysis of the $L_{2}$-discrepancy of symmetrisized point sets, ibid. 132 (2001), 1-18.

[4] - - - Sums of distances to the nearest integer and the discrepancy of digital nets, Acta Arith. 106 (2003), 379-408.

[5] G. Larcher and G. Pirsic, Base change problems for generalized Walsh series and multivariate numerical integration, Pacific J. Math. 189 (1999), 75-105.

[6] H. Niederreiter, Point sets and sequences with small discrepancy, Monatsh. Math. 104 (1987), 273-337.

[7] —, Random Number Generation and Quasi-Monte Carlo Methods, CBMS-NSF Regional Conf. Ser. in Appl. Math. 63, SIAM, Philadelphia, 1992.

Institut für Analysis

Universität Linz

Altenbergerstraße 69

A-4040 Linz, Austria

E-mail: friedrich.pillichshammer@jku.at

Received on 11.2.2002

and in revised form on 6.11.2002 